\title{
Verificação e calibração do modelo de simulação do desempenho reprodutivo de vacas de corte baseado no escore de condição corporal - avaliação interna
}

\section{José Acélio Silveira da Fontoura Júnior ${ }^{1^{*}}$, Frank Siewerdt², Nelson José Laurino Dionello ${ }^{3}$, Marcio Nunes Corrêa ${ }^{1}$, Carlos André de Oliveira da Silva ${ }^{4}$}

\footnotetext{
1 Programa de Pós-graduação - Universidade Federal de Pelotas.

2 Universidade de Maryland, USA.

${ }^{3}$ Universidade Federal de Pelotas. Bolsista de Produtividade do CNPq

${ }^{4}$ Universidade Federal do Pampa.
}

RESUMO - O trabalho foi desenvolvido com os objetivos de demonstrar e fazer a avaliação interna do modelo matemático desenvolvido para descrever o processo reprodutivo em sistemas de produção e simular o desempenho reprodutivo de vacas de corte a partir do escore de condição corporal ao parto (ECCPAR). O modelo é baseado na relação entre o ECCPAR e o intervalo parto-concepção (IPC) para vacas com cria ao pé; para novilhas e vacas vazias à concepção, foi baseado na probabilidade de ocorrência. Para a avaliação interna do modelo, foi utilizada a metodologia de verificação e calibração. O uso da técnica de variação dos dados de entrada foi feito por meio da construção de cenários, variando ECCPAR, data de início da estação reprodutiva (IER), data média do parto (DPAR) e ganho médio diário do nascimento ao desmame (GMDND). Esses cenários serviram também para demonstração do modelo. Por meio da calibração, foram escolhidos novos desvios-padrão para as variáveis: período de gestação, GMDND e data média do parto (DPAR). Os testes de degenerescência e independência de sementes geradoras de números aleatórios, após a calibração, comprovaram coerência do modelo na geração de aleatoriedade para as variáveis de interesse. A variação nos dados de entrada indicou eficácia do modelo para simular a dinâmica do processo reprodutivo, no entanto ajustes na taxa de concepção de primíparas são necessários para que o modelo simule valores compatíveis com a realidade.

Palavras-chave: bovinos de corte, modelagem, modelos matemáticos, reprodução

\section{Verification and calibration of a simulation model for reproductive performance of beef cows based on body condition score - internal evaluation}

\begin{abstract}
This work was carried out with the goal of demonstrating and performing internal evaluation of the mathematical model developed to describe the reproductive performance in production systems and to simulate reproductive performance of beef cows from the body condition score at calving (BCSAC). This model is based on the relationship between BCSAC and the interval between calving and conception (ICC) for lactating cows; for heifers and empty cows at conception, it was based on the probability of occurrence. For internal evaluation of the model, it was used the methodologies of verification and calibration. The use of technique of variation of input data was done based through the construction of scenarios by changing BCSAC, starting date of the reproductive season (SDRS), average date of calving (ADC), and average daily weight gain from birth to weaning (ADWGBW). These scenaries also served for demonstration of the model. Through calibration, it was chosen new standard deviations for the following variables: gestation period, ADWGBW and average birth date (ABD). Tests for degenerescence and independence of seeds generating random numbers, after calibration, showed coherence of the model in generating randomness for the variables being studied. Variation on the input data showed effectiveness of the model to simulate the dynamics of reproduction systems. Nevertheless, adjustments in conception rate of primiparous females are needed for the model to generate values that are compatible with reality.
\end{abstract}

Key Words: beef cattle, mathematical models, modeling, reproduction

Recebido em 20/3/2009 e aprovado em 2/12/2009.

Correspondências devem ser enviadas para: acelio.fontoura@unipampa.edu.br

*Endereço atual: Universidade Federal do Pampa. 


\section{Introdução}

A modelagem, processo de construção de um modelo, é, sobretudo, uma tentativa de integração de diferentes fenômenos, limitada pelos recursos humanos e materiais utilizados na sua construção (Lovatto \& Sauvant, 2001) e baseada principalmente na sistematização, o que permite o posicionamento do modelador frente ao estado de conhecimento (Lovatto, 2003). Modelar um sistema é entender seu funcionamento e poder prever o seu comportamento em diferentes condições (Fialho, 1999). Contudo, uma etapa importante no desenvolvimento de um modelo é sua avaliação, que se divide em interna e externa. A avaliação externa refere-se ao processo de validação do modelo, enquanto a interna refere-se à coerência no interior do modelo (Lovatto, 2003). A técnica de verificação procura garantir que o programa computacional que suporta o modelo esteja isento de erros (Freitas Filho, 2001).

Em um sistema pecuário, existem várias opções e interações entre técnicas de manejo e genética e as limitações de tempo e monetária para avaliar esses sistemas são fatores limitantes. A simulação por computador é uma importante ferramenta para avaliar sistemas pecuários (Tess \& Kolstad, 2000), portanto a avaliação, interna e externa, de modelos de simulação é fundamental. Os processos de avaliação podem usar testes estatísticos para comparar resultados simulados com resultados reais; no entanto, Harrison (1990) mostrou que testes estatísticos são inapropriados para validar modelos e sugeriu que testes subjetivos são mais úteis e confiáveis para avaliar o desempenho dos mesmos.

Um modelo teórico do desempenho reprodutivo de vacas de corte baseado no escore de condição corporal foi descrito por Fontoura Jr. et al. (2009) no intuito de desenvolver uma ferramenta para simular o desempenho reprodutivo e quantificar as mudanças no sistema de cria a partir de estratégias de manejo e/ou alterações das variáveis de entrada no modelo. $\mathrm{O}$ trabalho foi desenvolvido com os objetivos de demonstrar e fazer a avaliação interna do modelo matemático desenvolvido por Fontoura Jr. et al. (2009) para descrever o processo reprodutivo em sistemas de produção, bem como as simulações de desempenho reprodutivo feitas pelo modelo.

\section{Material e Métodos}

O modelo foi desenvolvido e implementado no software RURAL FAZPEC. Trata-se de um modelo empírico, dinâmico que contém elementos determinísticos e estocásticos. A descrição é feita de acordo com os modelos conceituais (Figuras 1 e 2) para vacas com cria ao pé e novilhas ou vacas solteiras, respectivamente. Os componentes do

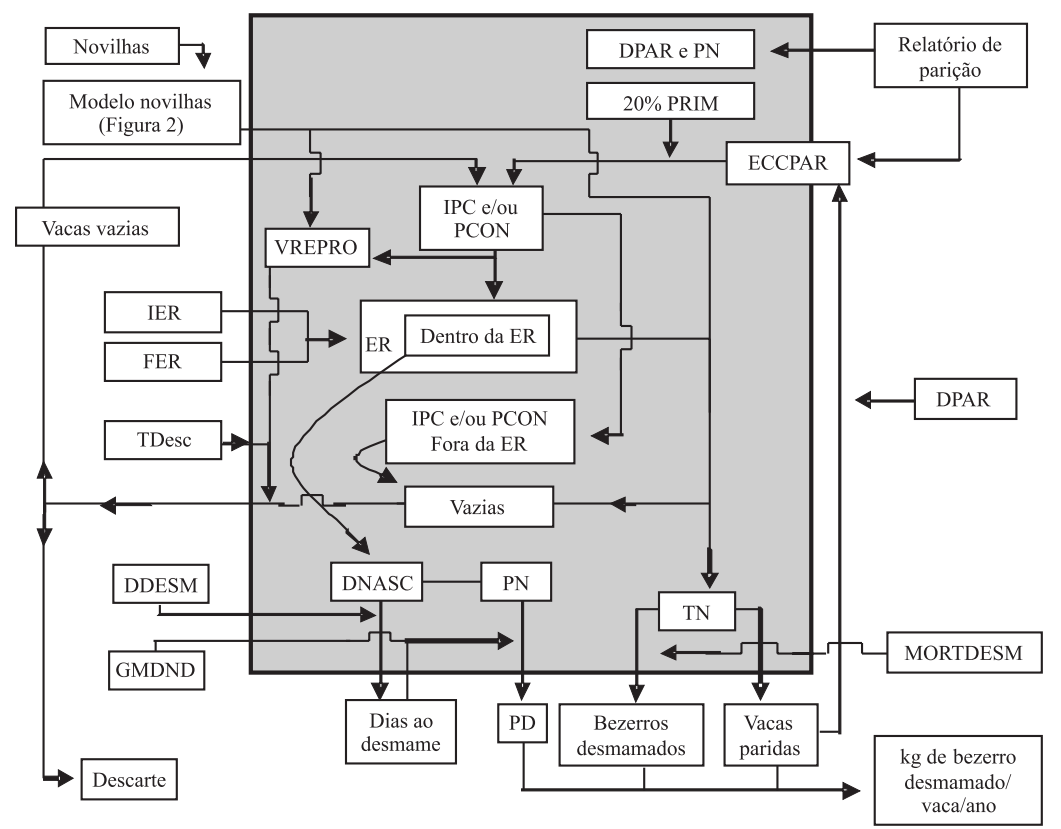

DPAR: data do parto; PN: peso ao nascimento; PRIM: primíparas ECCPAR: escore de condição corporal ao parto; IPC: intervalo parto concepção; PCON: probabilidade de concepção para vacas vazias e novilhas; VREPRO: vacas em reprodução; IER, FER e ER: início da, fim da e estação reprodutiva, respectivamente; TDesc: taxa de descarte; DDESM: data do desmame; GMDND: ganho médio diário do nascimento ao desmame; DNASC: data do nascimento; PD: peso ao desmame; TN: taxa de natalidade; MORTDESM: mortalidade do nascimento ao desmame.

Figura 1 - Modelo conceitual da vida reprodutiva, em quilogramas de bezerro desmamado, de vacas de corte a partir do escore de condição corporal (Fontoura Jr. et al., 2009). 


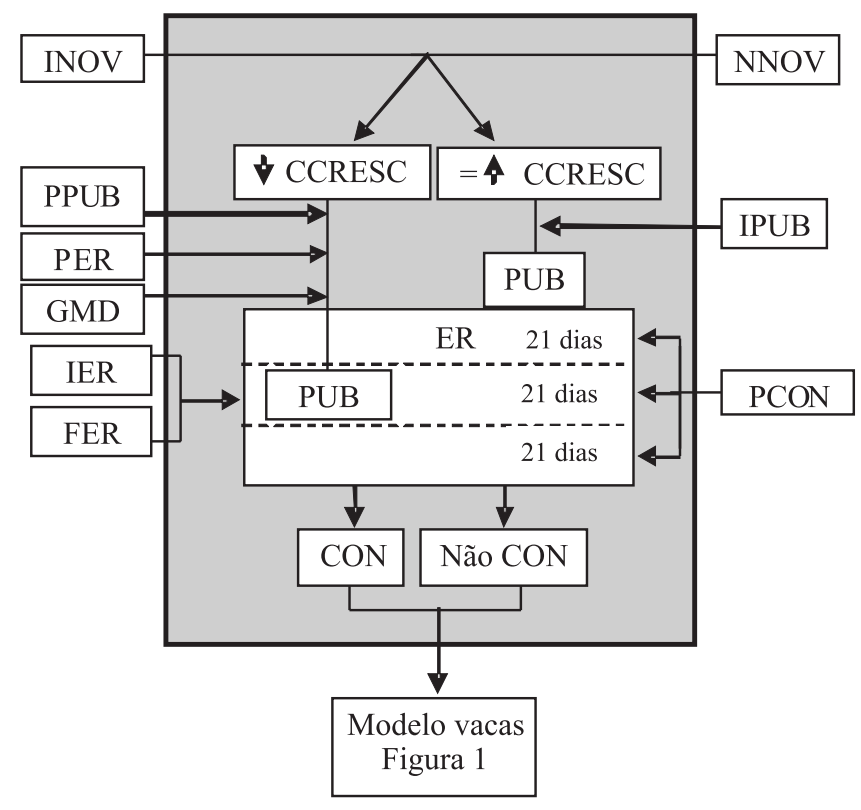

INOV = idade da novilha; NNOV = número de novilhas; CCRESC = curva de crescimento; PPUB = peso à puberdade; PER = peso da novilha ao início da estação reprodutiva; IER, FER e ER = início da, fim da e estação reprodutiva; $\mathrm{PUB}=$ puberdade; $\mathrm{PCON}=$ probabilidade de concepção; $\mathrm{COM}=$ concepção; IPUB = idade à puberdade.

Figura 2 - Modelo conceitual da concepção em novilhas baseado na curva de crescimento, idade da novilha, probabilidade de concepção, idade à puberdade e peso a puberdade (Fontoura Jr. et al., 2009).

modelo e suas formas de funcionamento foram descritos por Fontoura Jr. et al. (2009).

As metodologias adotadas para avaliação interna do modelo foram calibração e verificação. A calibração tem por finalidade promover ajustes de alguns parâmetros do modelo para que os resultados simulados tenham comportamento semelhante aos reais ou experimentais (Vargas et al., 2006). Já a verificação, considerando que modelos são construídos a partir de uma série de pressupostos e simplificações sobre o comportamento do sistema real, consiste em avaliar se esses pressupostos e simplificações foram corretamente implementados no modelo computacional. As técnicas de verificação adotadas neste trabalho foram: variações sobre os dados de entrada, emprego de testes de degenerescência e independência de sementes geradoras de números aleatórios (Freitas Filho, 2001). Já a calibração foi utilizada para avaliar os desvios-padrão utilizados quando empregada a aleatoriedade por meio de distribuições normais.

A seguir são descritas as técnicas de verificação segundo Freitas Filho (2001) e variáveis testadas para cada técnica: 1) Variação sobre os dados de entrada - consiste em alterar os dados de entrada do modelo e verificar se as respostas são adequadas e consistentes, pois variando os dados de entrada, espera-se que os resultados (saídas) sofram variações condizentes. Esse teste foi utilizado para avaliar o modelo de maneira geral, criando cenários (conforme abaixo) caracterizados por variações nos valores de entrada do modelo e, posterior, análise e discussão dos dados de saída do modelo. 2) Emprego de testes de degenerescência - consiste em verificar o funcionamento do modelo quando, sobre os parâmetros, aplicam-se valores extremos (menores ou maiores) que os permitidos. Esse teste foi usado para testar a coerência e eficiência de variáveis com valores de máximo e mínimo, sendo elas: DPAR, IPC mínimo, PN, GMDND. O intuito é gerar valores de acordo com os desvios-padrão propostos para cada variável e verificar se o modelo retorna valores que não extrapolem os limites impostos ao modelo. DPAR - os valores de máximo e de mínimo para essa variável devem ser, respectivamente, menor ou igual à data de FEP e maior ou igual à data de IEP, devendo os valores pertencer ao intervalo de estação de parição; IPC - o modelo deve levar em consideração o período de puerpério das vacas, sendo o valor de IPC maior que 42 dias; $\mathrm{PN}$ - os valores de máximo e mínimo para essa variável foram estabelecidos em 50 e $15 \mathrm{~kg}$, respectivamente (Gunski et al., 2001); GMDND - os valores de máximo e mínimo para essa variável foram estabelecidos em 1,6 e 0,150 kg/animal/dia, respectivamente (Teixeira \& Albuquerque, 2003). Para as variáveis PN e GMDND, foram estabelecidos esses limites no intuito de evitar erros, inclusive de digitação. 3) Independência de sementes geradoras de números aleatórios - as sementes geradoras de números aleatórios não devem influenciar o comportamento geral dos resultados. Diferenças nos valores retornados pelos geradores de números aleatórios, entre as diversas simulações, são esperadas, mas tais diferenças, considerando-se as médias dessas simulações, devem ser mínimas, caracterizando que estas são devidas aos processos aleatórios envolvidos no sistema real e bem representados no modelo. Para o modelo foram utilizadas distribuições normais e GNA (geradores de números aleatórios) para as seguintes variáveis: PN e DPAR (que é igual a DNASC) quando esses não forem lidos do relatório de parição e, ainda para IPC (valor aleatório somado ao resultado da equação que determina o IPC com base no ECCPAR), GMDND, duração da gestação e, somente para novilhas, PER. Para essa análise, foram confrontados os dados de uma simulação com os dados médios de 30 simulações, sendo um número total de 300 animais para cada simulação do modelo.

Os histogramas usados para os testes de calibração, emprego de testes de degenerescência e independência de sementes geradoras de números aleatórios foram feitos no programa R (R Development Core Team, 2007). 
Os cenários simulados foram construídos baseados na variação das variáveis de entrada do modelo. Para o ECCPAR foram utilizados três cenários que constaram da variação no ECCPAR, sendo: ECC2, ECCPAR igual 2, ECC3, ECCPAR igual a 3 e ECC4, ECCPAR igual a 4. As variáveis IER, ER, DPAR média, GMDND, DDESM, PN, duração da gestação e MORTDESM, \% de PRIM e PER foram mantidas fixas para os 5 anos e iguais a: 01/11/; 85; 10/10; 0,650, 15/04; 30, 282, 0, 20 e 260, respectivamente (Figura 3). Os outros cenários foram baseados na variação do IER, DPAR (média), GMDND e ECCPAR (Figura 4). Se todas essas variações fossem geradas ter-se-ia um total de 27 cenários, o que dificultaria a análise e interpretação dos resultados. Portanto, foram simulados em um primeiro momento os três cenários de variação de ECCPAR. O intuito inicial foi demonstrar, avaliar e verificar a consistência dos dados de saída do

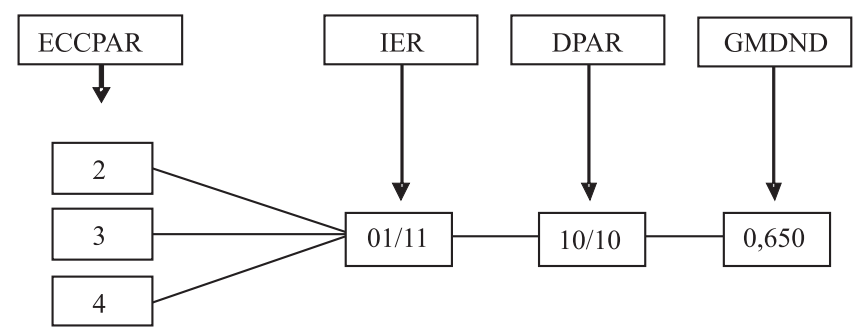

DPAR = data do parto; ECCPAR = escore de condição corporal ao parto; IER = início da estação reprodutiva, GMDND = ganho médio diário do nascimento ao desmame.

Figura 3 - Estrutura dos cenários montados para verificação do modelo, de acordo com a variação no escore de condição corporal ao parto.

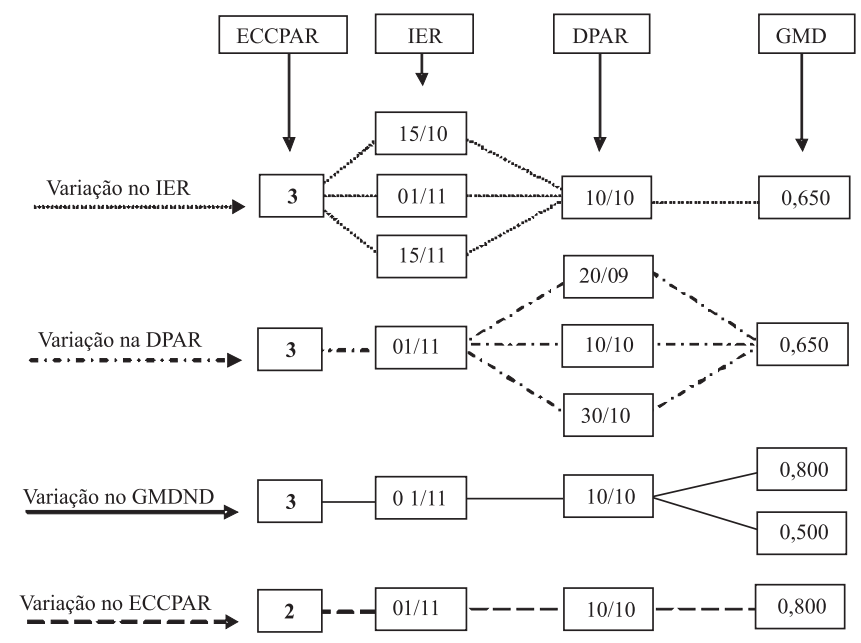

DPAR = data do parto; ECCPAR = escore de condição corporal ao parto; IER = início da estação reprodutiva, GMDND = ganho médio diário do nascimento ao desmame.

Figura 4 - Estrutura dos cenários montados para verificação do modelo. modelo de acordo com as variações no ECCPAR. Foi feita uma simulação para cada cenário e, para cada uma dessas, um número total de vacas igual a 500 foi utilizado. Posteriormente, foram testadas variações nas variáveis IER, DPAR, GMDND e ECCPAR, mantendo-se o ECCPAR constante para 8 cenários e ao longo dos cinco anos, $\mathrm{ECCPAR}=3$, exceto para um cenário que foi igual a 2 . Foram utilizadas três datas (cenários) para IER, 15/10, 01/11 e 30/ 11, sendo que a DPAR e GMDND foram mantidos fixos e iguais a 10/10 e 0,650 kg/animal/dia, respectivamente; três datas para DPAR média, 20/09, 10/09 e 30/10, mantendo-se o IER e o GMDND fixos e iguais a 01/11 e 0,650 kg/animal/ dia, respectivamente; e dois valores de GMDND (0,500 e $0,800 \mathrm{~kg} / \mathrm{animal} / \mathrm{dia}$ ), mantendo o IER e a DPAR fixos e com valores igual a 01/11 e 10/10, respectivamente. O único cenário que variou ECC, que foi igual a 2, utilizou IER igual a 01/11, DPAR igual a 10/10 e GMDND igual a 0,800 kg/ animal/dia. Os cenários foram denominados da seguinte maneira: 01/11,15/11 e 30/11 para variação no IER, 20/09, 10/10 e30/10 para DPAR, 500 e 800 para GMDNDeECC2(Figura 4).

A variação no ECCPAR teve como propósitos, com exceção do ECC2, os mesmos valores de entrada do cenário 800, avaliar e demonstrar o impacto dessa variável sobre os dados de saída. Nesse segundo momento, os objetivos foram demonstrar e verificar a consistência dos dados de saída de acordo com as variações nas variáveis mencionadas. Foram feitas 30 simulações para cada cenário com um número total de vacas igual a 300 .

Para o primeiro e segundo momentos, considerou-se a primeira estação de parição e estação de reprodutiva na primavera de 2007; desmame em abril de 2008 e próxima estação de parição na primavera de 2008.

A falta de dados reais que contemplem todas a variáveis de entrada e saída contempladas pelo modelo impossibilitou validar o modelo. No entanto, a mais útil avaliação do modelo refere-se à crítica da lógica, às referências de literatura e às suposições usadas na construção do modelo (Tess \& Kolstad, 2000).

\section{Resultados e Discussão}

Os resultados da calibração e do emprego dos testes de degenerescência e de independência de sementes geradoras de números aleatórios são apresentados nas Figuras 5, 6, 7, 8, 9 e 10 para as variáveis: período de gestação, PN, IPC, GMDND, DPAR/DNASC e PER, respectivamente.

Nos testes de calibração para período de gestão foram avaliados o DP = 2 dias proposto por Fontoura Jr. et al. (2009) e dois desvios-padrão maiores: 5 e 8 dias com $\bar{X}=282$ dias. Com base na análise da Figura 5A, por meio da calibração, 
foi redefinido o DP adotado no modelo conceitual (Fontoura Jr. et al., 2009), sendo o novo DP = 5 dias. Paschal et al. (1991) observaram período de gestação médio de 282 para Bos taurus e 292 dias para Bos indicus com desvios-padrão médios de 1,5 dia para essa variável. Os desvios-padrão encontrados para o período de gestação estão de acordo com os de Rocha et al. (2005), de 8,4 e 10,5 dias, para animais Nelore e Hereford-Nelore, respectivamente. Cavalcante et al. (2001) encontraram desvio-padrão de 3,65 dias e período de gestação de 284 dias para a raça Nelore. Rocha et al. (2005) encontraram ainda amplitude de variação de 40 dias para o período de gestação. Os dados simulados pelo modelo (Figura 5A) têm amplitude, para o $\mathrm{DP}=5$ dias, de 30 dias, para essa simulação do modelo. As considerações anteriores corroboram a mudança no desvio-padrão proposto por Fontoura Jr. et al. (2009), passando-se a adotar então o desvio-padrão de 5 dias. Houve coerência do modelo, visto que em 30 simulações as variações foram mínimas (Figura 5B), caracterizando que a maior amplitude de variação relativa a uma simulação é devida ao processo aleatório e caracteriza o sistema real.

Na calibração do desvio-padrão relativo à variável peso ao nascimento foram testados três diferentes desviospadrão: 1 , 2 e 3 kg, com $\bar{X}=30$ kg (Figura 6 A). O valor de 2 foi proposto por Fontoura Jr. et al. (2009). Para peso ao nascimento, foi confirmado o uso do desvio-padrão de $2 \mathrm{~kg}$. Lopes et al. (2008) encontraram para a raça Nelore desviospadrão de 2,96; 2,88 e 2,43 kg para diferentes ambientes (Rio Grande do Sul, Santa Catarina e Paraná, respectivamente).
Já o teste de degenerescência, para o desvio-padrão adotado, não acusou valores fora dos limites máximo e mínimo impostos para essa variável (Figura 6A), confirmando a viabilidade de uso do desvio-padrão adotado. Cabe ressaltar que foram testados, em uma primeira análise, desviospadrão de 5, 3 e $1 \mathrm{~kg}$, porém o uso de desvio-padrão igual a 5 extrapolou os limites mínimo e máximo para várias simulações do modelo, havendo valores maiores que 50 e menores que $15 \mathrm{~kg}$. Assim, decidiu-se rodar o modelo com outros valores de desvio-padrão (3, 2 e 1 kg).

$\mathrm{O}$ teste de independência de sementes geradoras de números aleatórios foi realizado com 1 e 30 simulações (Figura 6B). O desvio-padrão e $\bar{X}$ utilizados para construção da análise foram, respectivamente, 2 e $30 \mathrm{~kg}$. No histograma fica evidenciado o bom comportamento do modelo, visto que as variações em torno da média são maiores, quando se compara os valores de 1 com 30 simulações do modelo, visto que é esperada maior variância para um conjunto de dados do que para a média de 30 . Essa justificativa também é válida para os histogramas a seguir, referentes aos testes de independência de sementes geradoras de números aleatórios.

Em 7A foram testados três desvios-padrão 3, 5 e 7 dias com $\bar{X}=87$ dias. Foi adotado o valor de 5 dias, conforme Fontoura Jr. et al. (2009). O desvio-padão adotado caracteriza uma amplitude que interessa ao modelo no intuito de melhor representar a realidade, visto que valores de ECC diferentes podem ter, não frequentemente, o mesmo valor de IPC, o que é observado em sistemas de cria. Os histogramas das
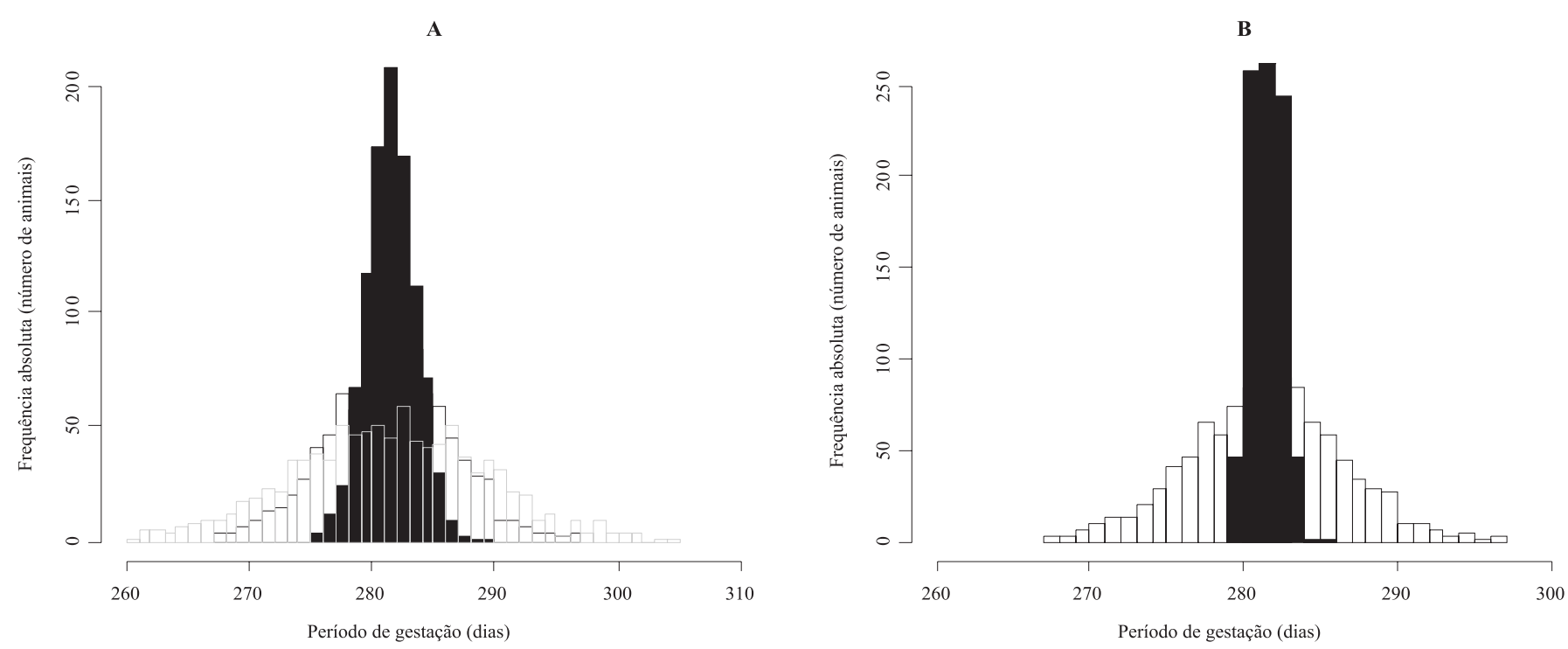

Em A: calibração e teste de degenerescência, com $\bar{X}=282$, e DP =2 (coluna preta), 5 (borda preta) e 8 (borda cinza); em B: teste de independência de sementes geradoras de números aleatórios, com $X=282$ e DP =5 para 1 simulação (bordas pretas) e 30 simulações (coluna preta).

Figura 5 - Período de gestação. 

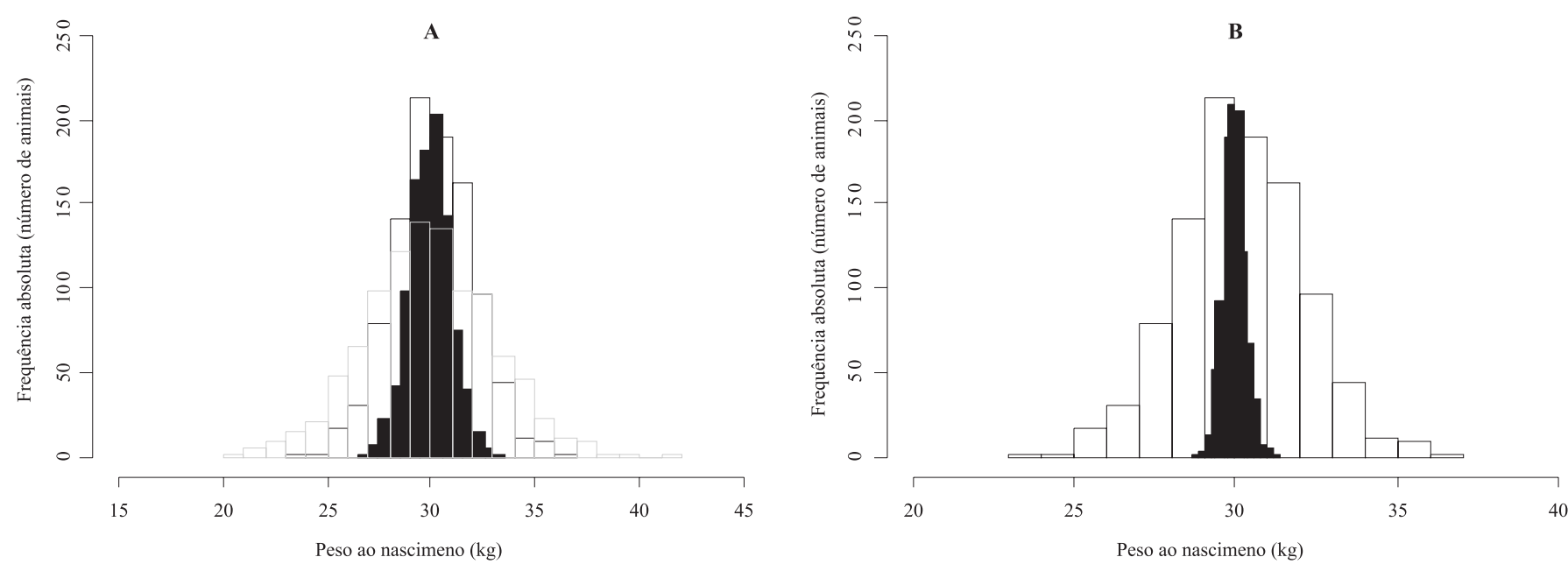

Em A: calibração e teste de degenerescência, $\operatorname{com} X^{-}=30$, e DP= 1 (coluna preta), 2 (borda preta) e 3 (borda cinza); em B: independência de sementes geradoras de números aleatórios, $\operatorname{com} X=30$ e DP $=2$ para 1 (bordas pretas) e 30 simulações (coluna preta).

Figura 6 - Peso ao nascimento.
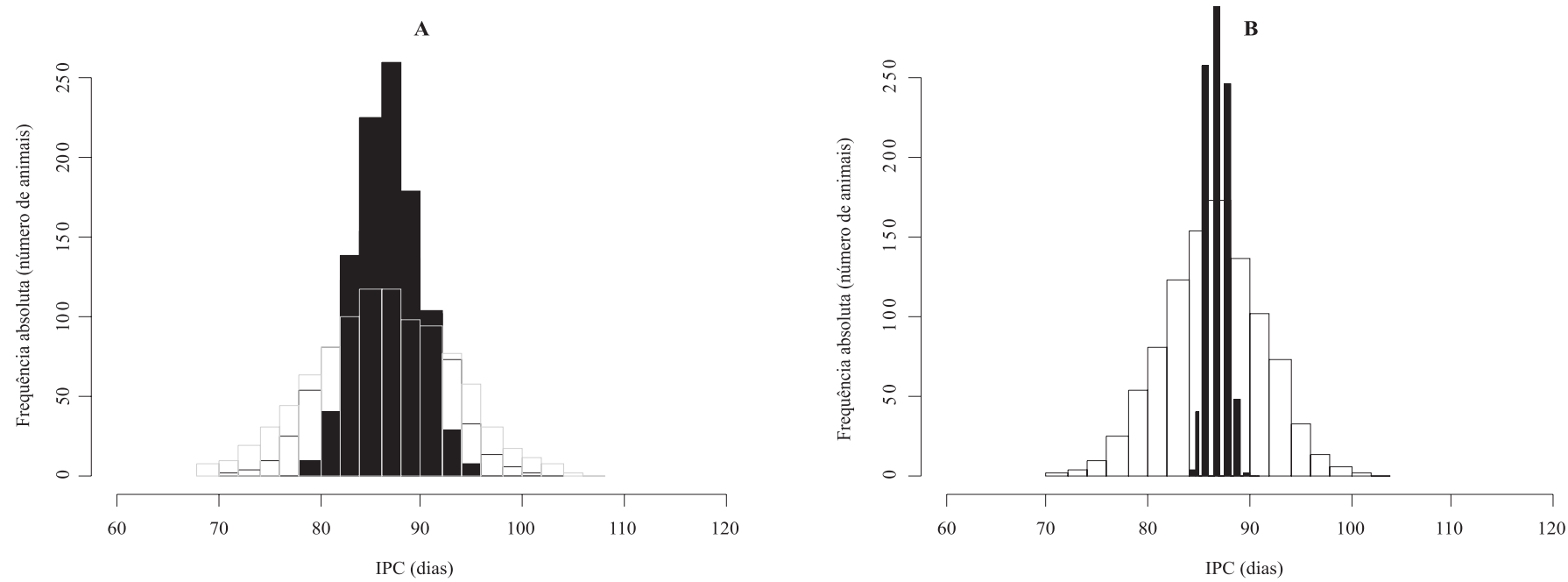

C
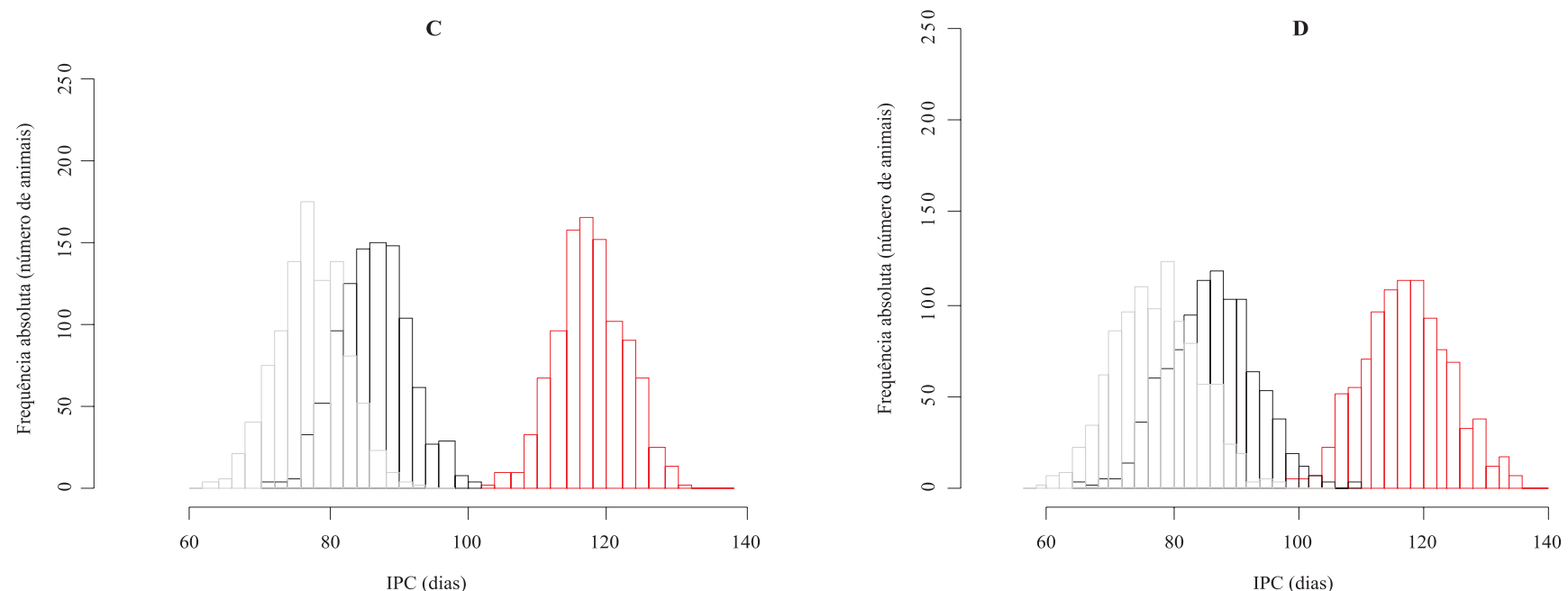

Em A: calibração e teste de degenerescência, desvio-padrão 3 (coluna preta), 5 (borda preta) e 7 (borda cinza); em B: independência de sementes geradoras de números aleatórios, para 1 (borda preta) e 30 simulações (coluna preta); em C: escore de condição corporal 2 (bordas vermelhas), 3 (bordas pretas) e 4 (bordas cinzas) para desviopadrão de 5; em D: idem C com exceção do desvio-padrão, que é de 7.

Figura 7 - Intervalo parto-concepção, em dias. 
Figuras 7C e 7D confirmam esse pressuposto, pois aplicado aleatoriedade para diferentes ECC (2, 3 e 4) os valores extremos de cada ECC são congruentes. A avaliação do desvio-padrão 7 (Figura 7D) teve como objetivo verificar qual desvio representa melhor o pressuposto de equivalência de valores para os limites extremos de cada ECC. A análise confrontada a Figura 7C contribuiu para a escolha do desvio de 5 , pois o de 7 dias gera um número muito grande de valores equivalentes para IPC para vacas com diferentes ECCPAR, o que não representa a variação existente nos sistemas de produção.

O histograma 7B mostra o teste de independência de sementes geradoras de números aleatórios com valores gerados por uma e 30 simulações do modelo, com $\bar{X}=87$ e $\mathrm{DP}=5$ dias. $\mathrm{O}$ comportamento do modelo foi adequado, pois as variações em torno da média foram maiores, quando comparados os valores de uma ou 30 simulações do modelo. A argumentação é a mesma referente aos histogramas 5B e $6 \mathrm{~B}$, no que tange o objetivo da técnica de verificação.

A Figura 8A mostra o histograma relativo à variável GMDND, em kg/animal/dia, com $\bar{X}=0,550$ e DP=0,025, 0,050 e $0,100 \mathrm{~kg}$. O desvio-padrão original proposto no desenvolvimento do modelo (Fontoura Jr. et al., 2009) foi de $0,100 \mathrm{~kg}$. No entanto, após análise do histograma 8A foi reconsiderado esse valor e feita a opção pelo $\mathrm{DP}=0,050$. Embora alguns autores relatem desvios-padrão maiores que o adotado no modelo, por exemplo, Teixeira e Albuquerque (2003) que relatam DP $=0,160 \mathrm{~kg} / \mathrm{dia}$, a análise dos histogramas 8C e 8D permitiu optar pelo uso do DP = 0,050 , visto que desvios menores $(0,025)$ não gerariam dados congruentes, o que, do ponto de vista do modelo, quanto a representação da realidade, não é interessante.
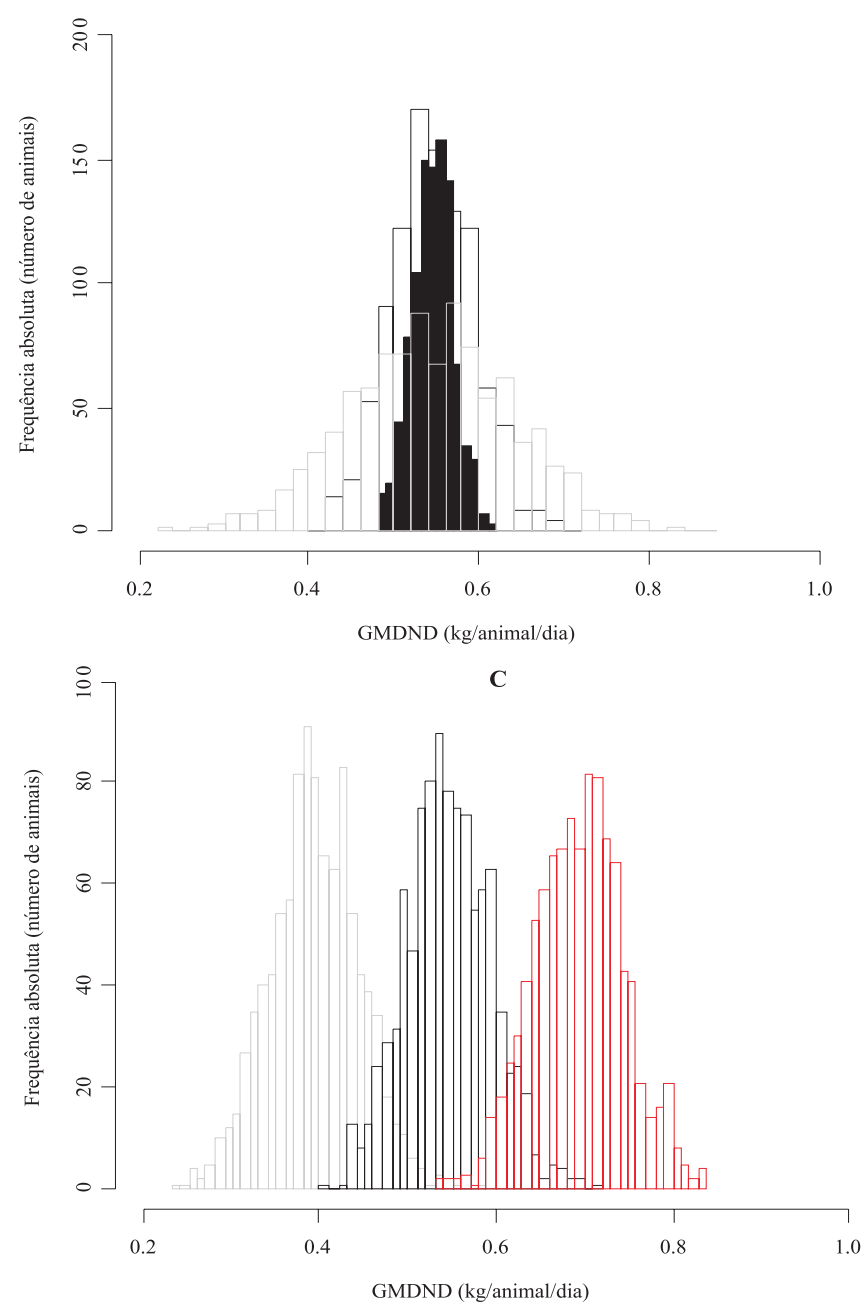
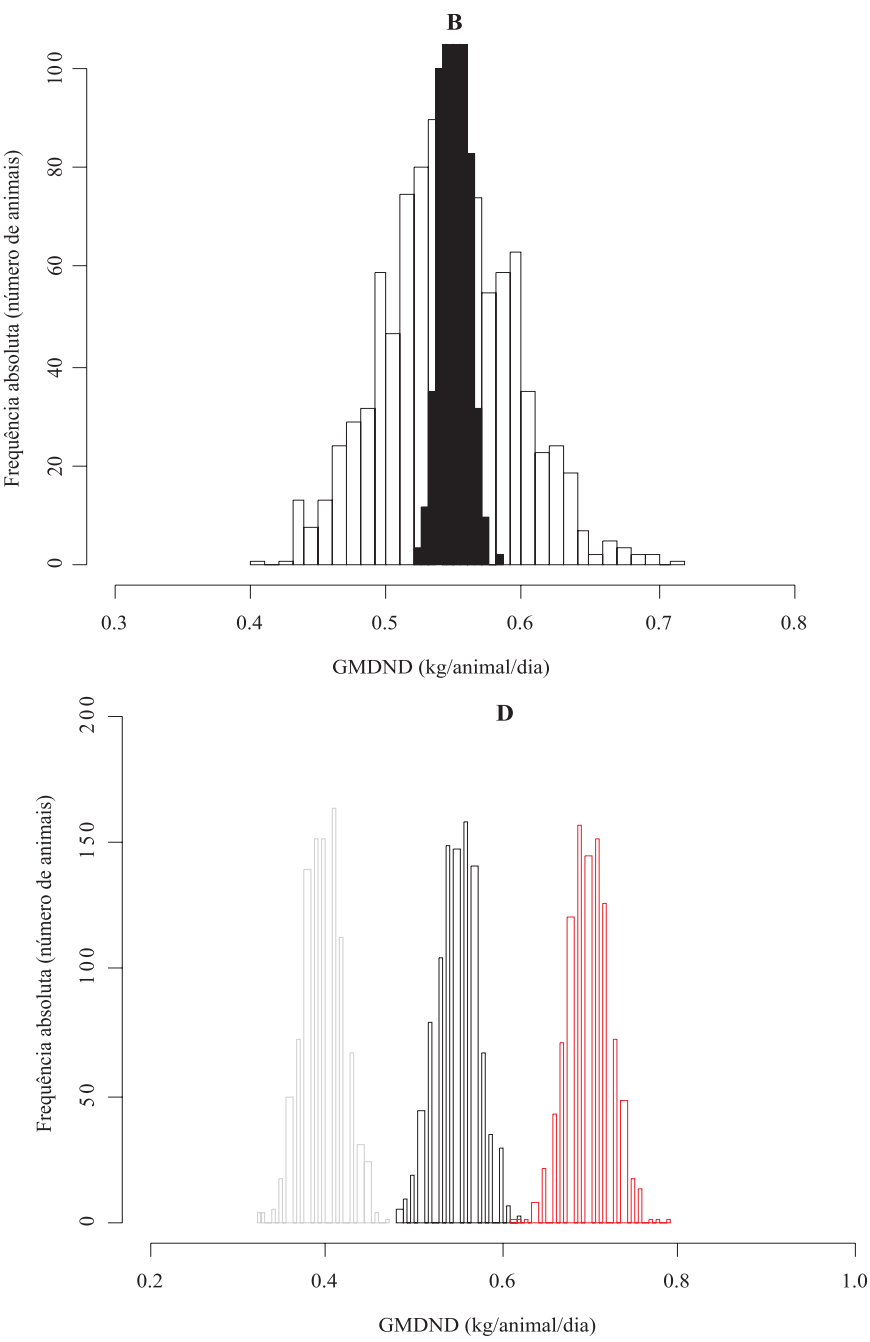

Em A: calibração e teste de degenerescência, $\bar{X}=0,550$ kg/animal/dia, e desvio-padrão de 0,025 (coluna preta), 0,050 (borda preta) e 0,100 (borda cinza); em B: independência de sementes geradoras de números aleatórios, $X=0,550$ e desvio-padrão de 0,050 para 1 simulação (bordas pretas) e 30 simulações (coluna preta); em C: variação no ganho médio diário do nascimento ao desmame de 0,400 (bordas cinzas), 0,550 (bordas pretas) e 0,700 (bordas vermelhas) para um desvio-padrão de 0,050 kg; e em D: idem C, com exceção do desvio-padrão, que é de 0,025 kg.

Figura 8 - Ganho médio diário do nascimento à desmana, em kg/animal/dia. 
Em 8B, no teste de independência de sementes geradoras de números aleatórios, foram confrontados os dados de uma contra 30 simulações do modelo, ambos com $\bar{X}=0,550$ e $\mathrm{DP}=0,050 \mathrm{~kg} / \mathrm{animal} / \mathrm{dia}$; conforme já discutido nos histogramas 5B, 6B e 7B, os dados seguiram o comportamento esperado para esse tipo de variável.

Na Figura 9A, encontram-se os testes de calibração e degenerescência para a variável DPAR/DNASC. A média foi considerada a DPAR média e o desvio-padrão um percentual do valor da estação reprodutiva, que para essa análise foi de 90 dias. Para a construção dos histogramas, a DPAR média foi considerada como dia zero. Foram utilizados desvios-padrão de 10, 20 e 30\%, o que corresponde em dias, para a estação reprodutiva considerada, a: 9, 18 e 27 dias, respectivamente. A Figura 9A mostra a inviabilidade de uso de desvio-padrão igual ou superior a $20 \%$, visto que esse extrapola os limites mínimos e máximos referentes à estação de parição. Com base nisso o modelo assume o DP de 10\%, como uma re-opção, através da calibração e teste de degenerescência, ao desvio-padrão proposto no modelo original (Fontoura Jr. et al., 2009). Em9B, a aplicação do teste de independência de sementes geradoras de números aleatórios, com $X^{-}=$DPAR média e $\mathrm{DP}=10 \%$, para 1 e 30 simulações. Conforme já discutido nos histogramas $5 \mathrm{~B}, 6 \mathrm{~B}, 7 \mathrm{~B}$ e $8 \mathrm{~B}$ os dados mostram o comportamento esperado para as simulações.

Com base na análise da Figura 10A, optou-se pelo uso de desvio-padrão 10, que se aproxima mais do caráter aleatório pretendido no modelo. No teste de independência de sementes geradoras de números aleatórios (Figura 10B), O modelo mostrou a mesma coerência já discutida para

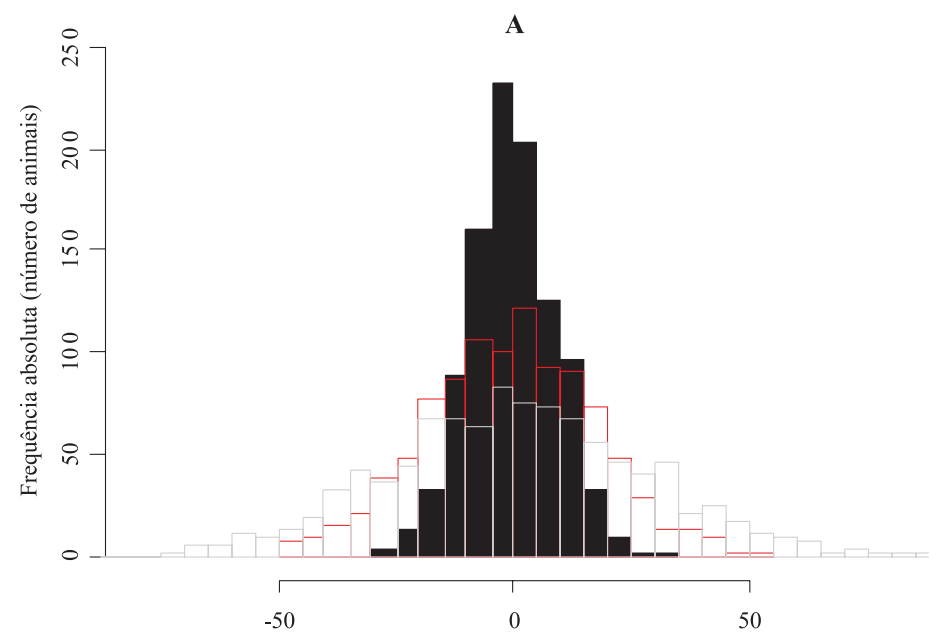

Data do nascimento/parto, desvios em relação a média (dias) outras variáveis em que foi aplicado o mesmo teste.

De maneira geral, o modelo teve comportamento satisfatório para as taxas de concepção para vacas, novilhas, primíparas, concepção geral e kg de bezerro desmamado por vaca por ano (Tabela 1). As taxas de concepção aumentaram conforme o ECCPAR, com a variação entre anos, para o cenário ECC2, esperada, pois a baixa concepção em um ano acarretará maior concepção no outro. Isto ocorre porque, nessa simulação, foi usado um número fixo de vacas ao longo dos anos, com reposição e descarte igual a 20\%. Como a taxa de concepção é baixa para o ECCPAR igual a 2, o modelo assume para o outro ano um dado número de vacas vazias. Essas terão taxas de concepção maior, o que conduzirá uma maior taxa de concepção geral para o dado ano.

O valor para taxa de concepção no ano 1, para o cenário ECC2, pode parecer baixo, no entanto, para essa simulação, é coerente, visto que o ECCPAR é igual 2. Além disso, o início da estação reprodutiva é $01 / 11$ e a data média do parto 10/10, havendo pouco tempo para recuperarem a condição corporal. Salienta-se que 10/10 é data média do parto, o que implica a existência de vacas parindo dentro da estação reprodutiva e com ECC 2. Ao considerar a taxa de concepção média para os três cenários (ECC ao parto 2, 3 e 4), para o primeiro ano, essa taxa é de 66\%, razoável para um rebanho com 33\% das vacas com ECC2.

As taxas de concepção para novilhas foram consistentes, visto que as novilhas entraram na ER com $10 \mathrm{~kg}$ abaixo do PPUB; caso o PER fosse igual ao PPUB no IER, essas taxas seriam maiores. Como o peso médio foi mantido fixo para todos os anos, as taxas foram semelhantes, com pequenas variações ocasionadas pelo caráter aleatório
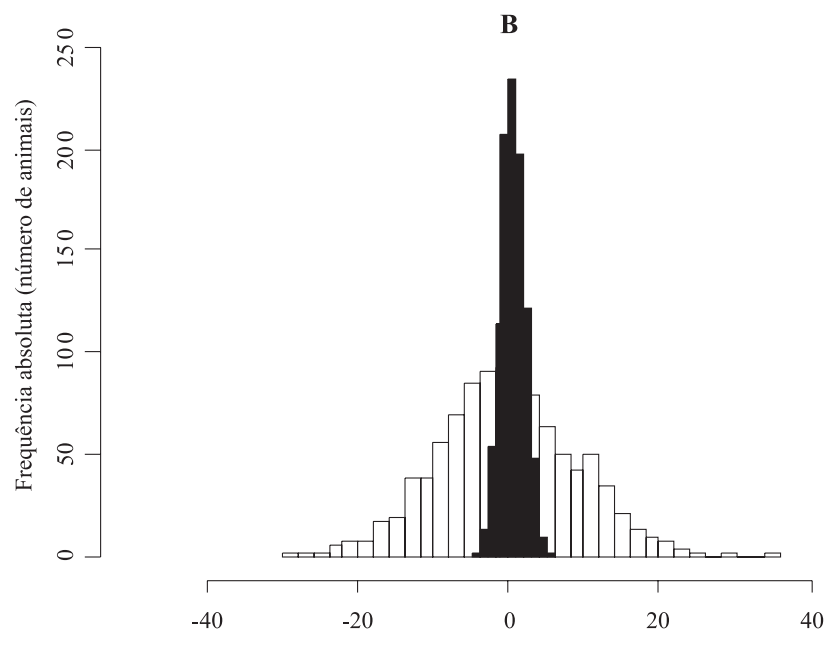

Data do nascimento/parto, desvios em relação a média (dias)

Em A: calibração e teste de degenerescência, $\bar{X}=$ data X (igual ao dia 0), e desvio-padrão de 10\% (coluna preta), 20\% (borda vermelha) e 30\% (borda cinza); e em B: independência de sementes geradoras de números aleatórios, com $\bar{X}=$ data X e DP $=10 \%$ para 1 simulação (bordas pretas) e 30 simulações (coluna preta).

Figura 9 - Data ao parto e data ao nascimento com desvios em dia em relação à data $\bar{X}$ de parto. 

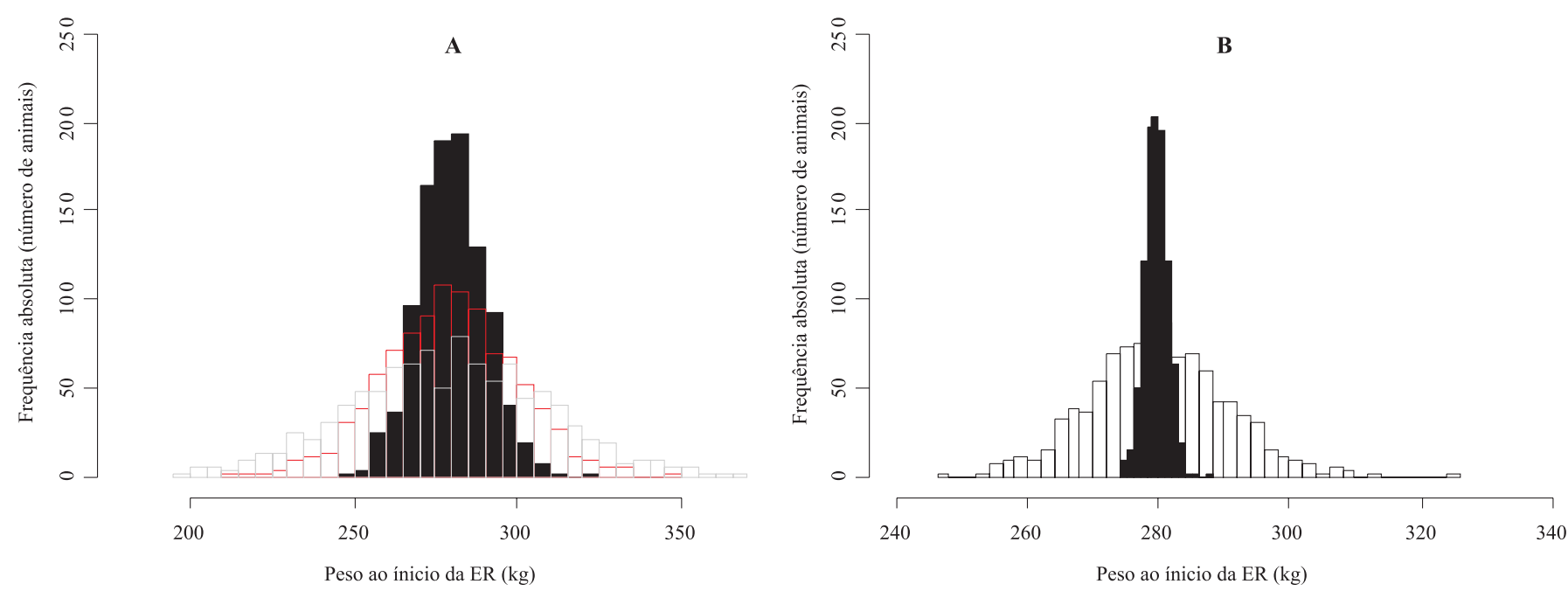

Em A: calibração e teste de degenerescência, peso $\bar{X}=280$, e DP = 10 (coluna preta), 20 (borda vermelha) e 30 kg (borda cinza); e em B: independência de sementes geradoras de números aleatórios, $\bar{X}=280$ e DP $=10 \mathrm{~kg}$ para 1 simulação (bordas pretas) e 30 simulações (coluna preta).

Figura 10 - Peso ao início da estação reprodutiva, em kg PV/animal.

Tabela 1 - Taxa de concepção e quilogramas de bezerro desmamado/vaca/ano para os cinco anos de simulação de acordo com a variação no escore de condição corporal ao parto

\begin{tabular}{|c|c|c|c|c|c|c|c|c|}
\hline \multirow[t]{2}{*}{ Cenário } & \multirow{2}{*}{$\begin{array}{c}\text { Taxa de } \\
\text { concepção }\end{array}$} & \multicolumn{6}{|c|}{ Anos } & \multirow{2}{*}{$\begin{array}{l}\text { kg de bezerro/ } \\
\text { vaca/ano }\end{array}$} \\
\hline & & 1 & 2 & 3 & 4 & 5 & Média & \\
\hline \multirow[t]{3}{*}{ Escore de condição corporal ao parto igual a 2} & Vacas & 5,8 & 71,7 & 43,2 & 19,9 & 42,3 & 37 & \\
\hline & Primíparas & 0,0 & 46,1 & 46,9 & 38,5 & 36,0 & 33 & \\
\hline & Geral & 16,5 & 69,0 & 49,6 & 34,0 & 46,5 & 43 & 75,0 \\
\hline \multirow[t]{2}{*}{ Escore de condição corporal ao parto igual a 3} & Vacas & 93,0 & 81,4 & 82,2 & 84,8 & 89,0 & 86 & \\
\hline & Geral & 77,8 & 78,7 & 80,1 & 83,5 & 87,0 & 81,4 & 116,8 \\
\hline \multirow[t]{4}{*}{ Escore de condição corporal ao parto igual a 4} & Vacas & 99,0 & 99,5 & 99,5 & 99,5 & 97,4 & 99 & \\
\hline & Novilhas & 75,0 & 82,0 & 84,0 & 76,0 & 81,0 & 80 & \\
\hline & Primíparas & 38,0 & 88,0 & 74,1 & 86,9 & 77,6 & 73 & \\
\hline & Geral & 84,8 & 94,7 & 92,7 & 93,5 & 91,4 & 91 & 129,8 \\
\hline
\end{tabular}

do PER, essas variações no peso são responsáveis pela oscilação na taxa de concepção.

As taxas de concepção para prímiparas é um ponto a ser revisto no modelo, já que estão superestimadas, com exceção do ano 1, para os cenários 2 e 3, que tiveram taxas subestimadas. Mesmo assim, a concepção em primíparas é coerente no que se refere ao ECCPAR, sendo maiores à medida que o ECCPAR aumenta. Cabe ressaltar que as taxas de concepção para primíparas nos cenários 3 e 4, embora teoricamente superestimadas, para esses valores de ECC, raros em sistemas reais para essa categoria, devem realmente ser altas. O ponto que deve ser re-calibrado é magnitude desse aumento. No entanto, o aumento é esperado quando se aumentam os ECCPAR, sobretudo para essa categoria. A recalibração será feita com base na análise de sucessivas simulações.

O aumento nas taxas de concepção, para os cenários 3 e 4, ao longo dos anos, é coerente, pois como se trata de ECC altos, os IPC são baixos e as vacas vão, a medida que avançam os anos, tendo partos menos tardios e aumentam a probabilidade de concepção.

A saída $\mathrm{kg}$ de bezerros desmamados/vaca/ano teve um comportamento adequado sendo maior à medida que foi aumentado o ECCPAR das vacas. Esse aumento encontra suporte na maior taxa de concepção, consequentemente maior número de bezerros desmamados, pois o modelo não considera o GMDND como fator dependente do ECCPAR. 
De acordo com os dados de saída na variação no IER, houve coerência e eficácia do modelo na simulação. As variáveis dias ao desmame, PD não sofrem variação com a data IER, o que segue a lógica proposta pelo modelo, pois essas variáveis não são influenciadas pela data IER. Já variáveis como IPC e IEP sofreram variações pequenas, o que é bastante coerente, tendo em vista a maior probabilidade de concepção quando se usam IER mais tardios, o que ocasiona maior TCON (ver TCON, Tabela 2), contudo com pequenos aumentos no IPC e no IEP, devido à possibilidade de concepções mais tardias. A necessidade de atrasar o IER ou aumentar o período de ER é destacada por Werth et al. (1991), no sentido de incrementar a taxa de prenhez em vacas de corte.

As variações na DPAR (média) também tiveram coerência nos dados de saída. Ao contrário do cenário anterior, houve diminuições nos valores de saída para dias ao desmame, PD, IPC, IEP e TCON à medida que a DPAR média elevou. Para as variáveis relacionadas ao desmame, quando o parto é mais tardio, espera-se que os bezerros sejam desmamados com menor idade (data de desmame foi mantida fixa) e menor peso. A diminuição no IPC e IEP também é esperada em DPAR mais tardias e, embora o IPC seja menor (média das vacas que conceberam), a taxa de concepção decresce com o avanço da DPAR pelo fato de o IER ter sido mantido fixo para as três datas de parto, o que faz com que algumas vacas estejam aptas a conceber, somente após o término da estação reprodutiva. O IEP diminuiu porque a data do próximo parto se torna mais tardia com o atraso na DPAR do ano corrente. A comparação dos cenários com variação no GMDND (500 e 800) reforça a coerência do modelo, pois apenas os dados de saída referentes ao peso à desmama sofreram variação, o que era esperado. As demais variáveis mantiveram-se constantes em relação à variação do GMDND, respeitando a lógica de desenvolvimento do modelo. A última saída do modelo, referente ao cenário ECC2, foi com intuito de mostrar o impacto gerado pela mudança de 1 ponto no ECC. Cabe ressaltar que esse cenário tem os mesmo dados de entrada, com exceção do ECC, do cenário 800. Mas, os dados de saída de IPC, IEP e TCON são bastante inferiores, do ponto de vista

Tabela 2 - Resultados de data ao parto, peso ao nascimento, dias e peso ao desmame, intervalo parto concepção, data da concepção e do próximo parto e taxa de concepção para os cenários simulados

\begin{tabular}{|c|c|c|c|c|c|c|c|c|c|}
\hline Cenário & $\begin{array}{l}\text { Data do } \\
\text { parto } \\
\text { (data) }\end{array}$ & $\begin{array}{c}\text { Peso ao } \\
\text { nascimento } \\
\text { (kg) }\end{array}$ & $\begin{array}{c}\text { Dias ao } \\
\text { desmame } \\
\text { (dias) }\end{array}$ & $\begin{array}{c}\text { Peso ao } \\
\text { desmame } \\
\quad(\mathrm{kg})\end{array}$ & $\begin{array}{c}\text { Intervalo } \\
\text { parto-concepção } \\
\text { (dias) }\end{array}$ & $\begin{array}{l}\text { Início da } \\
\text { estação } \\
\text { (dias) }\end{array}$ & $\begin{array}{c}\text { Data da } \\
\text { concepção }\end{array}$ & $\begin{array}{l}\text { Data do } \\
\text { parto } \\
\text { próximo }\end{array}$ & $\begin{array}{c}\text { Data da } \\
\text { concepção } \\
\text { (\%) }\end{array}$ \\
\hline $1 / 11$ & $9 / 10 / 2007$ & 29,9 & 188 & 152,3 & 86 & 368 & $1 / 1 / 2008$ & $8 / 10 / 2008$ & 65,4 \\
\hline $15 / 11$ & $9 / 10 / 2007$ & 29,9 & 188 & 152,2 & 89 & 370 & $6 / 1 / 2008$ & $13 / 10 / 2008$ & 86,5 \\
\hline $31 / 11$ & $9 / 10 / 2007$ & 29,9 & 188 & 152,1 & 92 & 373 & $9 / 1 / 2008$ & $16 / 10 / 2008$ & 95,9 \\
\hline $20 / 9$ & $20 / 9 / 2007$ & 30,0 & 207 & 164,8 & 92 & 374 & $21 / 12 / 2007$ & 28/9/2008 & 98,1 \\
\hline $10 / 10$ & $11 / 10 / 2007$ & 29,9 & 187 & 151,1 & 89 & 371 & 7/1/2008 & $15 / 10 / 2008$ & 86,5 \\
\hline $30 / 10$ & $28 / 10 / 2007$ & 29,9 & 170 & 140,4 & 86 & 367 & $20 / 1 / 2008$ & $27 / 10 / 2008$ & 63,5 \\
\hline 0,500 & $11 / 10 / 2007$ & 30,1 & 186 & 123,4 & 89 & 370 & $7 / 1 / 2008$ & $14 / 10 / 2008$ & 85,2 \\
\hline 0,800 & $11 / 10 / 2007$ & 30,1 & 186 & 179,4 & 89 & 370 & $7 / 1 / 2008$ & $14 / 10 / 2008$ & 85,4 \\
\hline ECC2 & $9 / 10 / 2007$ & 29,9 & 188 & 180,5 & 115 & 396 & $24 / 1 / 2008$ & $31 / 10 / 2008$ & 23,7 \\
\hline
\end{tabular}

Cenários: 1/11, 15/11 e 31/11/2007 datas do iníco da estação reprodutiva; 20/09, 10/10 e 30/10/2007 data média do parto; 0,500 e 0,800 ganho médio diário do nascimento ao desmame, em kg; ECC2 - escore de condição corporal igual a 2 ao parto.

zootécnico, para o cenário ECC2, o que mais uma vez confirma a estabilidade e coerência do modelo, na simulação dos dados de desempenho reprodutivo.

O valor de IPC médios, para os cenários com ECC fixos (3), num total de 240 simulações do modelo, foi de 89 dias, valor que está de acordo com os encontrados por Morrison et al. (1999), que foi de 87 dias para vacas com ECCPAR entre 5 e 6 e Richards et al. (1986), que encontraram 84 dias para vacas com ECC $\geq 5$ (escala de 1 a 9 , o valor 5 corresponde a 3 em uma escala de 1 a 5).

\section{Conclusões}

O modelo é coerente na geração de aleatoriedade para as variáveis de interesse e eficaz na simulação da dinâmica do processo reprodutivo, no entanto, ajustes na taxa de concepção de primíparas são necessários para que o modelo simule valores compatíveis com a realidade para essa variável. O modelo descreve adequadamente a eficiência do sistema de cria como função do escore de condição corporal ao parto, portanto, 
seu uso para fins didáticos pode contribuir para o processo ensino-aprendizagem. Os conceitos aplicados na construção desse modelo podem contribuir no desenvolvimento de outros modelos em estudos futuros em modelagem de sistemas de produção de bovinos de corte.

\section{Referências}

CAVALCANTE, F.A.; MARTINS FILHO, R.; CAMPELLO, C.C. et al. Período de gestação em rebanho Nelore na Amazônia Oriental. Revista Brasileira de Zootecnia, v.30, n.5, 2001, p.1451-1455.

FIALHO, F.B. Sistemas de apoio à decisão na produção de suínos e aves. In: REUNIÃO ANUAL DA SOCIEDADE BRASILEIRA DE ZOOTECNIA, 36., 1999, Porto Alegre. Anais... Porto Alegre: SBZ, 1999. p.307-317.

FONTOURA JR., J.A.S.; SIEWERDT, F.; DIONELLO, N.J.L. et al. Modelo teórico do desempenho reprodutivo de vacas de corte baseado no escore de condição corporal. Revista Brasileira de Zootecnia v.38, n.8, p.1627-1635, 2009.

FREITAS FILHO, P.J. Introdução à modelagem e simulação de sistemas, com aplicações em arena. Florianópolis: Visual Books, 2001. p.322.

GUNSKI, R.J.; GARNERO, A.V.; BEZERRA, L.A.F. et al. Idade ao primeiro parto, período de gestação e peso ao nascimento na raça nelore. Ciência Agronômica, v.32, n.1/2, p.46-52, 2001.

HARRISON, S.R. Regression of a model on real system output: an invalid test of model validity. Agricultural Systems, v.34, p.183-190, 1990.

LOPES, J.S.; RORATO, P.R.N.; WEBER, T. et al. Efeito da interação genótipo $\mathrm{x}$ ambiente sobre o peso ao nascimento, aos 205 e aos 550 dias de idade de bovinos da raça Nelore na Região Sul do Brasil. Revista Brasileira de Zootecnia, v.37, n.1, p.54-60, 2008.

LOVATTO P.A. Princípios de modelagem e sua aplicação no estudo de cadeias de produção agrícola. In: REUNIÃO ANUAL DA SOCIEDADE BRASILEIRA DE ZOOTECNIA, 40., 2003, Santa Maria. Anais... Santa Maria: 2003. (CD-ROM).
LOVATTO, P.A.; SAUVANT, D. Modelagem aplicada aos processos digestivos e metabólicos do suíno. Ciência Rural, v.31, n.4, 2001.

MORRISON, D.G.; SPITZER, J.C.; PERKINS, J.L. Influence of prepartum body condition score change on reproduction in multiparous beef cows calving in moderate body condition. Journal of Animal Science, v.77, p.1048-1054, 1999.

PASCHAL, J.C.; SANDERS, J.O.; KERR, J.L. Calving and weaning characteristics of Angus-, Gray Brahman-, Gir-, Indu-Brazil-, Nellore-, and Red Brahman-sired F1 calves. Journal of Animal Science, v.69, p.2395-2402, 1991.

R Development Core Team [2007]. R: A language and environment for statistical computing. R Foundation for Statistical Computing. Vienna, Austria. Disponível em: <http:/ /www.R-project.org>. Acesso em: 15/3/2008.

RICHARDS, M.W.; SPITZER, J.C.; WARNER, M.B. Effect of varying levels of pospartum nutrition and body condition at calving on subsequent reproductive performance in beef cattle. Journal of Animal Science, v.62, n.2, p.300-306, 1986

ROCHA, J.C.M.C.; TONHATI, H.; ALENCAR, M.M. et al. Componentes de variância para o período de gestação em bovinos de corte. Arquivo Brasileiro de Medicina Veterinária e Zootecnia, v.57, n.6, p.784-791, 2005.

SIGTECH TECNOLOGIA EM GESTÃO E UNIVERSIDADE FEDERAL DE PELOTAS - UFPel [2005]. Rural Fazpec software de gestão em pecuária. Disponível em: <http:// www.aphnet.com.br $>$ e $<$ www.ufpel.edu.br/nupeec $>$. Acesso em: 18/11/2008.

TEIXEIRA, R.A.; ALBUQUERQUE, L.G. Efeitos ambientais que afetam o ganho de peso pré-desmama em animais Angus, Hereford, Nelore e mestiços Angus-Nelore e Hereford-Nelore. Revista Brasileira de Zootecnia, v.32, n.4, p.887-890, 2003.

TESS, M.W.; KOLSTAD, B.W. Simulation of cow-calf production system in a range environment: I. Model development. Journal of Animal Science, v.78, p.1159-1169, 2000.

VARGAS, G.D.; DIONELLO, N.J.L.; BRUM, P.A.R. et al. Modelagem do crescimento e do desenvolvimento de frangos de corte: validação. Ciência Rural, v.36, n.5, p.1664-1669, 2006.

WERTH, L.A.; AZZAM, S.M.; NIELSEN, M.K. et al. Use of a simulation model to evaluate the influence of reproductive performance and management decisions on net income in beef production. Journal of Animal Science, v.69, p.4710-4721, 1991. 